\title{
Back-Reaction of Clocks and Limitations on Observability in Closed Systems
}

\author{
Aharon Casher* and Benni Reznik ${ }^{\dagger}$ \\ School of Physics and Astronomy, Tel Aviv University, Tel Aviv 69978, Israel
}

September, 2, 1999

\begin{abstract}
Measurements are ordinarily described with respect to absolute "Newtonian" time. In reality however, the switching-on of the measuring device at the instance of the measurement requires a timing device. Hence the classical time $t$ must be replaced by a suitable quantum time variable $\tau$ of a physical clock. The main issue raised in this article is that while doing so, we can no longer neglect the backreaction due to the measurement on the clock. This back-reaction yields a bound on the accuracy of the measurement. When this bound is violated the result of a measurement is generally not an eigenvalue of the observable, and furthermore, the state of the system after the measurement is generally not a pure state. We argue that as a consequence, a sub-class of observables in a closed system cannot be realized by a measurement.
\end{abstract}

\footnotetext{
*ronyc@post.tau.ac.il

†reznik@post.tau.ac.il
} 


\section{Introduction}

The special role of time in quantum theory has been noted since the early days of quantum mechanics. Unlike other observables time remains a classical variable. It cannot be a simply "quantized" because as is well known, for bounded Hamiltonians, a formal construction of a time operator gives rise to a non self-adjoint operator [1]. Nevertheless this difficulty becomes a serious obstacle, only when a system must be quantized as a whole, that is without first splitting between what is quantum and what is classical. In this case, since the total energy of a closed system is conserved, all observables become independent of the "external" classical time. Time must therefore be constructed "internally" from quantum variables alone. The conceptual as well as technical difficulties in carrying out this task are sometime referred to as 'The problem of time' in quantum theory [2, 3, 4, 5, 6, 7].

The aim of the present article is to examine the problem of time from the point of view of von-Neumann's quantum measurement theory [8]. In this theory, von-Neumann handles both the system and the measuring device on the same quantum mechanical footing, and provides a framework consistent with the following fundamental quantum postulates:

1. There is no fundamental restriction on the accuracy that one can measure a single observable.

2. The result of a precise measurement is one of the eigenvalues of the Hermitian operator representing the observable.

3. The state of the system after the measurement is "reduced" to the corresponding eigenstate. A precise measurement is hence also a preparation of a pure state.

von Neumann's theory handles time as a classical variable. In reality however the switching-on of the measuring device at the instance of the measurement requires a timing device. Hence the classical time $t$ must be replaced by a suitable quantum time variable $\tau$ of a physical clock [9, 3].

\footnotetext{
${ }^{1}$ In von Neumann's framework the initial state of the system is not (effectively or truly) reduced to the measured component as stated in 2 . and 3 above. It only shows that the correct correlation between a measuring device observable and the system can in principle be established. Issues concerning the interpretation or the reality of the reduction are not considered in this article, nor are they relevant to the conclusions pointed out here.
} 
The main issue raised in this article is that while doing so, we can no longer neglect the back-reaction due to the measurement on the clock. We will argue that:

1'. There is a fundamental restriction on the accuracy we can measure a single observable:

$$
\frac{\Delta J}{J} \geq \frac{\hbar}{\left(E_{C}-E_{0}\right) \delta T}
$$

Here, $\Delta J$ is the accuracy in a measurement of $J, E_{C}$, the clock energy is bounded from bellow by $E_{0}$, and $\delta T$ is the duration of the measurement.

We shall see that for a given clock energy, certain states of the system will only approximately satisfy postulates 2 . and 3 . above. However for other states which evolve sufficiently fast with respect to the time scale $\hbar /\left(E_{C}-E_{0}\right)$ the previous postulates are clearly violated:

2 '. The result of a measurement is generally not an eigenvalue of the observable.

3'. The state of the system after the measurement is generally not an eigenstate of the observable, nor will it be a pure state.

In fact the purity of the state of the system after the measurement is at best a good approximation. Generally after reduction to a certain value of the measuring device "pointer", the system remains correlated with the clock, i.e. it is in a mixed state. If we further measure the state of the clock and by that reduce the system into a pure state, the state of the system will not be correlated with the result of the measurement.

Why does von-Neumann's theory fail when the timing device is quantized? There are two separate reasons for this breakdown. First, due to the time-energy uncertainty relation, there is a minimal inaccuracy, and the time of the measurement becomes "fuzzy" by $\Delta \tau \geq \hbar / \Delta E_{C}$. Consequently, even if we can measure with arbitrary accuracy, the time of the measurement is still unknown by $\Delta \tau$. If during this time the system evolves considerably the final state of the system after the measurement is still correlated with the clock.

The second feature is the back-reaction on the clock [9, 10, 11]. When the measuring device becomes too accurate, i.e. $\Delta J / J<1 / E_{C} \delta T$, the backreaction on the clock increases and the clock's "trajectory" is distorted. It 
no longer shows the correct interaction time. For even better accuracy the clock is mostly "reflected back" in its coordinate time $\tau$. The phenomena is reminiscent to unitarity violations found in the WKB approach to time in quantum gravity [12, 13]. We will show that even for the minor part of measurements in which the clock is not reflected, the result still may not be interpreted as a successful measurement, since the required correlations between the measuring device and the relevant observable's eigenvalues are absent.

We will proceed as follows: In section 2. we first consider a measurement with an ideal timing device, i.e. the case that the clock Hamiltonian is unbounded. In this case the back-reaction does not effect the measurement. We show that observables defined "relative" to the clock time commute with the total Hamiltonian, in accordance to the "evolving constants of motion" approach of Rovelli [14, 15]. In section 3. we replace the ideal clock by a physical clock and derive the consequent bound (11). An exactly solvable toy model is used in section 4 . to examine in some details the breakdown of von Neumann's theory in the impulsive limit. In section 5. we conclude with some general remarks. In the appendix we review the basic properties of the "free particle clock" used in sections 3 and 4 . Throughout the article we choose units such that $\hbar=1$.

\section{Ideal Clocks in a Closed System}

The main issue of the article is to examine the effects of timing measurements by physical clocks. To relate our approach and result to the work already done on the "problem of time", we begin by first considering the case of a quantum measurement with an ideal clock in a closed system. By an ideal clock we refer to the unphysical case of a clock with unbounded energy. If for instance the Hamiltonian of the clock is linear

$$
H_{C}=c P_{x}
$$

the coordinate $x$ can be used to define the clock time $\tau=x / c$. As we now

show, the measurement problem, can be in this case well formulated relative to the clock time $\tau$.

Consider a closed system which includes an ideal clock and a measuring device, and consider a measurement of some observable $J$ at some internal 
time $\tau=\tau_{0}$. The full Hamiltonian is

$$
H_{\text {Closed }}=H_{C}+H_{M D}(Q, P)+H(J, \beta)+g\left(\tau-\tau_{0}\right) Q J
$$

where $\beta$ stands for other degrees of freedom of the system. For an ideal clock, $\tau$ is conjugate to the clock Hamiltonian which may be represented as $H_{C}=$ $-i \partial / \partial \tau$. The last term describes a standard von-Neumann measurement of an observable $J$ at the clock time $\tau=\tau_{0}$. For simplicity, and without loosing generality, we will next take $H_{M D}$, the Hamiltonian of the measuring device, be arbitrarily small.

Since $H_{\text {Closed }}$ is independent of the external time $t$, and since the system is closed, we may as well assume the closed system to be in an eigenstate of energy $E=E_{0}$,

$$
H_{\text {Closed }}|\Psi\rangle=E_{0}|\Psi\rangle
$$

As function of $\tau$ we get

$$
|\Psi(\tau)\rangle=\exp \left(-i\left(\tau-\tau_{i}\right) E_{0}-i Q \int_{\tau_{i}}^{\tau} g\left(\tau^{\prime}\right) J d \tau^{\prime}\right)\left|\Psi\left(\tau_{i}\right)\right\rangle
$$

Let us compare the correlations between the measuring device pointer variable, $P$, and the observable $J$ when $\tau_{i}<\tau_{0}$ and when $\tau>\tau_{0}$. In the impulsive limit $g \rightarrow \delta\left(\tau-\tau_{0}\right)$ the dynamical effect of $H(J, \beta)$ can be neglected and the measuring device pointer is shifted when $\tau>\tau_{0}$ by the value of $J$. Thus, if for $\tau<\tau_{0}$, there are no correlation between the measuring device and the system, for $\tau>\tau_{0}$ the correlations are of the same form as in an ordinary von-Neumann measurement. We also note that the shift in $P$ is independent of the particular total energy $E_{0}$ which affects only the overall phase.

It is often stated that non-trivial time dependent observables cannot be measured in a closed system. The basic argument goes like that: The Hilbert space of states describing a closed system is made of all states which are degenerate eigenstates of the total Hamiltonian. By the degeneracy any general state in this Hilbert space is also time independent. Therefore, only time independent operators, which commute with $H_{C l o s e d}$, constitute the set of observables for a closed system. Notice that this argument is valid for a bounded as well as unbounded Hamiltonian.

In our example above, since $H(J, \beta)$ is arbitrary, and since $\tau$ is conjugate to $H_{C}$ we have

$$
\left[J, H_{\text {Closed }}\right]=[J, H(J, \beta)] \neq 0
$$


and

$$
\left[\tau, H_{\text {Closed }}\right]=i
$$

Hence both the clock-time $\tau$ and the observable that we measure are according to this no-go theorem, non measurable observables!

To resolve this apparent contradiction we notice that in a closed system, only relative observables may be measured [16]. As is well-known, observables as position, velocity, angular momentum, etc, both in classical mechanics as well as in quantum mechanics, are relative observables. Indeed, we never measure the absolute position of a particle, but the distance in between the particle and some other object. The relative location, $r=x_{1}-x_{2}$ of two particles does commute with the total momentum $p=p_{1}+p_{2}+\ldots$. Similarly, we never measure the angular momentum of a particle along an absolute axis, but along a direction defined by some other physical objects. Therefore the angular momentum of a closed system can be measured only with respect to a point within the system, say the location of the center of mass, and along a direction defined by constitutes of the system. The absolute value of an observable is meaningless, and we have to relate this value to a 'reference system'.

In analogy, a time dependent observable, $J(t)$, cannot be measured at time $t$, because $J(t)$ does not commute with the Hamiltonian. However, we argue that any observable may be measured at an arbitrary time defined by means of an internal clock.

Suppose as an example we take $J=X$ of a free particle with $H=P_{X}^{2} / 2$, and consider a measurement at internal time $\tau_{0}$. The observable we measure is thus

$$
\hat{X}\left(\tau_{0}\right)=\hat{X}-\hat{P}\left(\hat{\tau}-\tau_{0}\right)
$$

To avoid confusion, for the rest of this section we will add to operators a 'hat', therefore $\hat{\tau}$ is an operator, while $\tau_{0}$ is a real number. Since the value of $\hat{X}$ at a certain instance does not change in time indeed

$$
\left[\hat{X}\left(\tau_{0}\right), H_{\text {Closed }}\right]=0
$$

Likewise, from the point of view of an external observer, $\hat{X}\left(\tau_{0}\right)$ is again a constant of motion that can be measured at any external time $t$. The internal clock time, $\hat{\tau}$, and $t$ are linearly related by $\hat{\tau}(t)=t+\hat{\tau}(0)$. Therefore, the external time $\hat{t}_{0}$ of the measurement is $\hat{t}_{0}=\tau_{0}-\hat{\tau}(0)=t-\left(\hat{\tau}(t)-\tau_{0}\right)$ Using the latter relation we can re-express $X\left(\tau_{0}\right)$ in terms of the external time $t$ as

$$
\hat{X}\left(\tau_{0}\right)=\hat{X}\left(\hat{t}_{0}\right)=\hat{x}(t)-\hat{p}\left(\hat{\tau}-\tau_{0}\right)
$$


Since $\left[x(t)-\hat{p}\left(\hat{\tau}-\tau_{0}\right), H_{\text {Closed }}\right]=0$ this is again a constant of motion. Notice that since $\hat{t}_{0}$ is an operator the time of the measurement remains uncertain with respect to $t$.

To summarized, our model suggests that the "time dependent" observables are in fact constant of motions. They are measured with respect to physical clock time and correspond to eigenstates of "composed" clocksystem operators as in eq. (10). More generally the observables are of the form

$$
\hat{J}\left(\tau_{0}\right)=U\left(\hat{\tau}-\tau_{0}\right) \hat{J}(t) U^{\dagger}\left(\hat{\tau}-\tau_{0}\right)
$$

For each real number $\tau_{0}$ we obtain another constant of motion.

Our discussion above seems to support the view suggested by Rovelli 14, 15]. According to him, the evolution in time of an observable is pictured as "evolving constants of motion". Indeed, in our case, a sequel of measurements of the same operator $A$, at different clock times $\tau_{0 i}$, yields a correlation between the "pointer" coordinate $\hat{P}_{i}$, of measuring device $i$, and the corresponding constants of motions $\hat{J}\left(\tau_{i}\right)$.

\section{Back-Reaction}

By assuming that clock Hamiltonian is unbounded, we have avoided the possibility of back-reaction on the clock due to the measurement. In the previous section, the trajectory of the time variable $\tau$ was linearly correlated to the external time irrespective of the coupling function $g(\tau)$. Since stable physical systems must have a ground state, we next reformulate the measurement problem using clocks with bounded energy. The general properties of physical clocks are described in the Appendix, where we also present the "free particle" toy model for a clock with a Hamiltonian $H_{C}=P_{X}^{2} / 2 M$. If the average momentum of the particle is much larger than the momentum uncertainty, $\left\langle P_{X}\right\rangle \gg \Delta P_{X}$, we can use as an approximate time variable the operator

$$
\tau \equiv \frac{X}{\left\langle P_{X}\right\rangle / M}, \quad\left[\tau, H_{C}\right]=i+O\left(\frac{\Delta P_{X}}{\left\langle P_{X}\right\rangle}\right)
$$

A Hamiltonian describing a measurement of an observable $J$, is hence

$$
H=\frac{P_{X}^{2}}{2 M}+H(J, \beta)+g(X) Q J
$$


The coupling function, $g(X)$, will be taken to be non-vanishing only in a finite region $-X_{0} / 2<X<X_{0} / 2$.

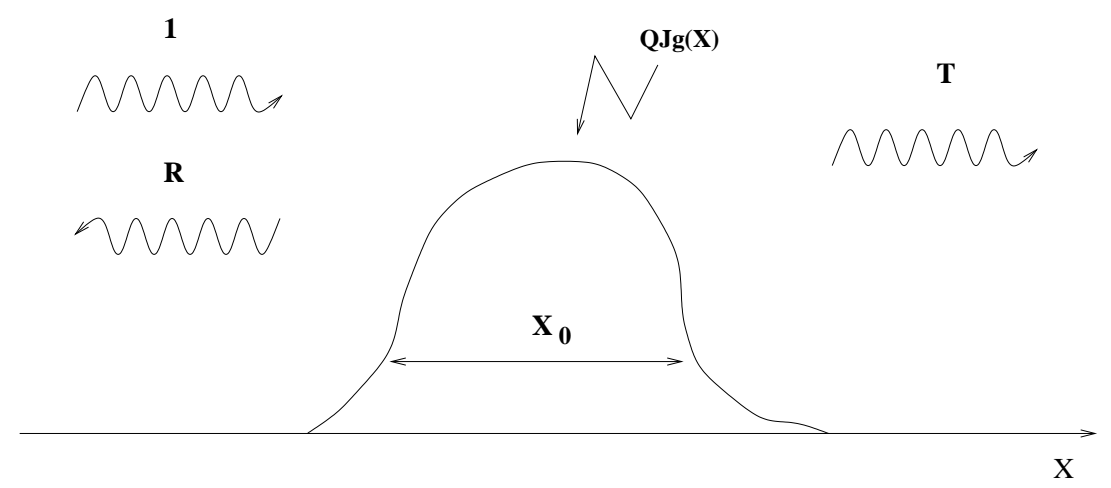

Figure 1: The effective potential of the clock, $\operatorname{Qjg}(X)$, is plotted as a function of the "time" coordinate $X$. At $X<X_{0}$ we must include back scattered (left moving) clock states.

There are two sources which contribute to the the uncertainty in the time of the measurement. The first is the quantum uncertainty $\Delta \tau$ (eq. (41) in the appendix). This quantum fuzziness of time is determined by the time-energy uncertainty relation

$$
\Delta \tau \Delta E \geq 1
$$

where $\Delta E$ is the uncertainty in the clock kinetic energy, and $\Delta \tau$ is the uncertainty associated with the uncertainty in the clock coordinate $X$.

The other source of uncertainty is due to the non-impulsive nature of $g(X)$. We will denote the latter by $\delta T$. If the relation (12) between $\tau$ an $X$ is still respected, we have

$$
\delta T \simeq \frac{X_{0}}{\left(\left\langle P_{X}\right\rangle / M\right)}
$$

However "during" the measurement (i.e. for $\left.X \in\left(-X_{0} / 2, X_{0} / 2\right)\right)$, the relation between $X$ and $t$ may be modified and the latter equation should be modified accordingly.

Quantum and classical measurements differ in one important respect. Only classically can we make the disturbance to the system arbitrarily small. 
In particular, since the accuracy of the measurement is proportional to $\Delta P \approx$ $1 / Q$, the strength of the interaction always scales like $\sim 1 / \Delta J$, i.e. it diverges for $\Delta J \rightarrow 0$.

In our case this interaction also affects the clock. According to the interpretation of $X$ as proportional to time, initially the clock wave function must be localized at $X \ll 0$ and after the measurement at $X \gg 0$. Therefore a "successful" measurement can be viewed as a scattering experiment for the clock, with only little back-scattered waves. However, an obvious consequence of the discussion above, it that in the limiting case $\Delta J \rightarrow 0$, the clock wave function will be mostly reflected back. Unlike the ordinary case where the accuracy determines only the disturbance caused to non-commuting observables, in our case the measurement affects the chance that an event of a measurement actually happens. A related "back-scattering" effect was previously discussed for in the case of a measurement of time of arrival [17, 7, [10].

To obtain a qualitative relation between the relevant scales in the problem: the accuracy of the measurement, the clock energy and accuracy, and the duration of the measurement let us examine the condition for very small back reaction. A sufficient requirement is that the kinetic energy $E_{C}$ of the clock be large enough with respect to the interaction term

$$
E_{C} \gg g_{0} Q J
$$

where $g_{0}$ is the average value of $g(X)$. This ensures that back-scattering effect is small and that during the measurement the clock's motion remains free up to small corrections. Therefore, up to corrections of order $O\left(\Delta P_{X} /\left\langle P_{X}\right\rangle\right)$, the eigenvalue equation $H \Psi=0$ for the closed system becomes

$$
i \frac{\partial \Psi}{\partial \tau}=(H(J, \beta)+g(X(\tau) Q J) \Psi
$$

where $\tau$ is given by (12). Hence the shift of the pointer variable is

$$
P_{M D}(\tau \gg 0)-P_{M D}(\tau \ll 0)=\int J g(X(\tau)) d \tau \approx g_{0} X_{0} \frac{M}{\left\langle P_{X}\right\rangle} \bar{J}
$$

were $\bar{J}=\int g J d \tau / \int g d \tau$, is the time average of $J$ over the time $\delta T$. We can now obtain a relation between the $\Delta P_{M D}$ of the "read out" measuring device coordinate and the accuracy $\Delta J$ of the measurement

$$
\Delta P_{M D} \simeq g_{0} X_{0} \frac{M}{\left\langle P_{X}\right\rangle} \Delta J\left(1+\frac{\bar{J}}{\Delta \bar{J}} \frac{\Delta P_{X}}{\left\langle P_{X}\right\rangle}\right)
$$


The last term, due to the uncertainty in $P_{X}$, contributes an additional uncertainty to the measurement. It may be interpreted as resulting from the uncertainty in the time of the measurement $\Delta \tau$. If the back-reaction is small, as in our case, this error is controlled by the time-energy uncertainty relation. As will be shown bellow we may neglect this term by taking sufficiently large $\left\langle P_{X}\right\rangle$.

Therefore we obtain the relation

$$
\Delta Q \geq \frac{\left\langle P_{X}\right\rangle}{g_{0} X_{0} M} \frac{1}{\Delta \bar{J}}
$$

Since we wish to minimize the right hand side of (16), we set $\langle Q\rangle=0$. We hence obtain the inequality

$$
\frac{\Delta \bar{J}}{\bar{J}} \gg \frac{1}{E_{C} \delta T} \approx \frac{1}{X_{0}\left\langle P_{X}\right\rangle}
$$

For measurements with accuracy $\Delta J / J=\epsilon$, the duration of the measurement must satisfy $\delta T \gg\left(\epsilon E_{C}\right)^{-1}$. The exact limit $\epsilon \rightarrow 0$ can be satisfied only with $E_{C} \rightarrow \infty$. We further note that the above inequality implies that $\frac{J}{\Delta J} \frac{\Delta P_{X}}{\left\langle P_{X}\right\rangle} \ll X_{0}\left\langle P_{X}\right\rangle$. Hence the approximation used in obtaining (20) is justified in for sufficiently large $\left\langle P_{X}\right\rangle$.

The inequality (21) is satisfied for time averaged observables. Therefore, the measured value will determine the state of the system as one of the eigenvalues only if $J$ is nearly constant during the time $\delta T$. Otherwise after the measurement the system remains in a mixed state. Let us denote by $\omega$ the typical frequency of $J(\omega \approx\langle[J, H(J, \beta)]\rangle /\langle J\rangle)$. We hence need to satisfy

$$
1 / \omega \gg \delta T \gg 1 / E_{C}
$$

or $\omega \ll E_{C}$. Therefore, only if $\omega$ is much smaller then the clock frequency $1 / E_{C}$, can we determine the state of the system after the measurement. It follows that the state of the subsystem with the highest energy available can not be measured.

\section{The impulsive limit}

In non-relativistic quantum mechanics there is no fundamental restriction on the duration, $\delta T$, of a measurement. The possibility of taking the limit 
$\delta T \rightarrow 0$ implies in principle the measurability at an instant of time. In the previous section we have argued that a necessary condition for an accurate measurement is that $E_{C} \delta T \gg 1$. We shall now examine in more details the consequences when this inequality is violated.

For concreteness we consider a solvable model for a measurement described, as before, by the interaction

$$
g(X) J Q
$$

where the observable $J$ is for simplicity we taken commute with the Hamiltonian $H(J, \beta)$. The coupling function is

$$
g(X)=\frac{\lambda}{X_{0}}\left(\theta\left(X-\frac{X_{0}}{2}\right)-\theta\left(X+\frac{X_{0}}{2}\right)\right)
$$

and the clock Hamiltonian is taken as before to be a free particle of mass $M$.

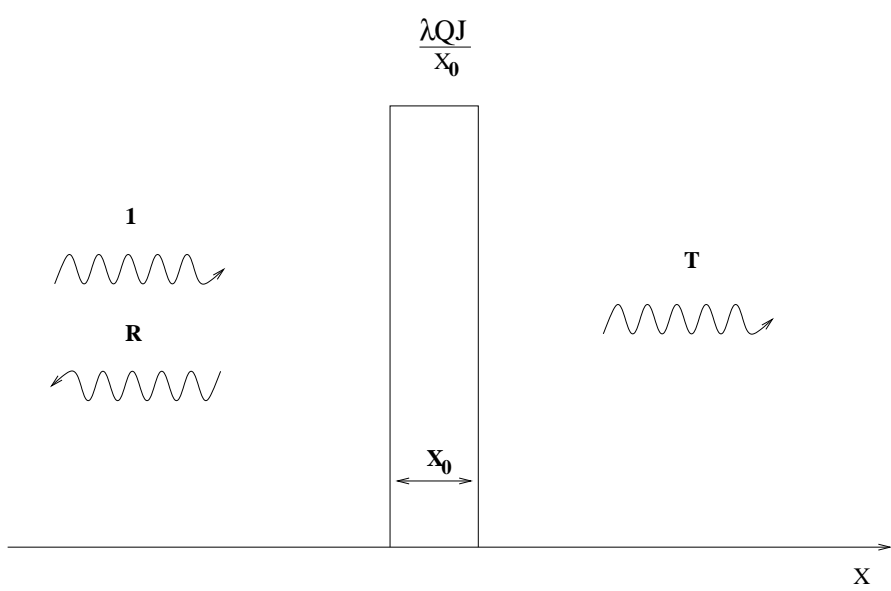

Figure 2: The effective potential in the impulsive limit $X_{0} \rightarrow 0, \int g(X) d X=$ $\lambda$

Let the initial state of the system be a direct product of the clock state $|C\rangle$, the measuring device state $|M D\rangle$ and the state of the system $|S\rangle$ :

$$
|\Psi\rangle_{I}=|C\rangle|M D\rangle|S\rangle
$$


for $X<0$. Then the transmitted wave function at $X>0$ is given by

$$
\left.|\Psi\rangle_{T}=\sum_{j} C_{j} \int d k \phi_{C}(k) T(k, Q, j)\right) e^{i k X}|k\rangle|M D\rangle|j\rangle
$$

Here $\phi_{C}(k)=\langle k \mid C\rangle$ is the clocks wave-function in the free momentum representation $k^{2}=2 M E_{C}$, and $C_{j}=\langle j \mid S\rangle$.

In the present case, the clock rate is affected during the interaction. For $|X|<X_{0}$, the momentum is given by $q^{2}=2 M\left(E_{C}^{0}+\lambda Q j / X_{0}\right)$. For sufficiently large $Q / X_{0}$ this momentum is completely dominated by the interaction rather than by the free momentum $k$. Hence the duration of the interaction is now

$$
\delta T \simeq M X_{0} / q
$$

The condition (21) will therefore be violated if

$$
E_{C} \delta T=q X_{0}<1
$$

where $E_{C}=q^{2} / 2 M$. The limit $q X_{0} \ll 1$, corresponds to the impulsive limit. In this case the analysis is greatly simplified. The transmission amplitude becomes

$$
T(k) \simeq \frac{1}{1+i \alpha Q} \quad \alpha \equiv \frac{\lambda j M}{k}
$$

In this limit we can therefore evaluate the shift of the measuring device pointer variable $P$ for a the case of transmitted clock state. Let us set for the initial state of the measuring device in (26), a Gaussian wave $\langle Q \mid M D\rangle \equiv$ $\chi_{M D}(Q)=\exp \left(-Q^{2} \Delta^{2} / 4\right)$. The accuracy of the measurement is therefore given by $\Delta$. The final wave function of the measuring device in the pointer representation can be evaluated to

$$
\begin{array}{r}
\chi_{M D}(P)=\int d Q e^{i Q P} \frac{1}{1+i \alpha Q} e^{-Q^{2} \Delta^{2} / 4}= \\
\frac{1}{\sqrt{4} \alpha}\left(1-\operatorname{erf}\left(\frac{1}{2} \frac{\Delta^{2}-2 \alpha P}{\Delta \alpha}\right)\right) \exp \left(-\frac{P}{\alpha}+\frac{\Delta^{2}}{4 \alpha^{2}}\right)
\end{array}
$$

To clarify the meaning of the last equation let us examine the final state of the measuring device in two limiting cases: when the disturbance caused to the clock wave function is small, i.e. the transmission amplitudes is nearly unity $T \simeq 1$, and the second case that of a strong back-reaction on the clock: $T \ll 1$ 
In the first case $|T| \simeq 1$ requires

$$
\frac{g_{0} j Q M}{k}=\alpha Q \approx \frac{\alpha}{\Delta} \ll 1
$$

Therefore we have

$$
T \simeq \exp (i \alpha Q)
$$

Substituting $T$ back into (26) we find that the shift caused to the measuring device pointer is

$$
\delta P_{M D}=\alpha=\lambda \frac{M}{k} j
$$

It is indeed proportional to the observable eigenvalues $j$. However, since by the uncertainty relation $Q \sim \Delta Q \geq 1 / \Delta P_{M D}$, the "weakness" condition (31) restricts the accuracy and yields

$$
\frac{\Delta j}{j} \gg 1
$$

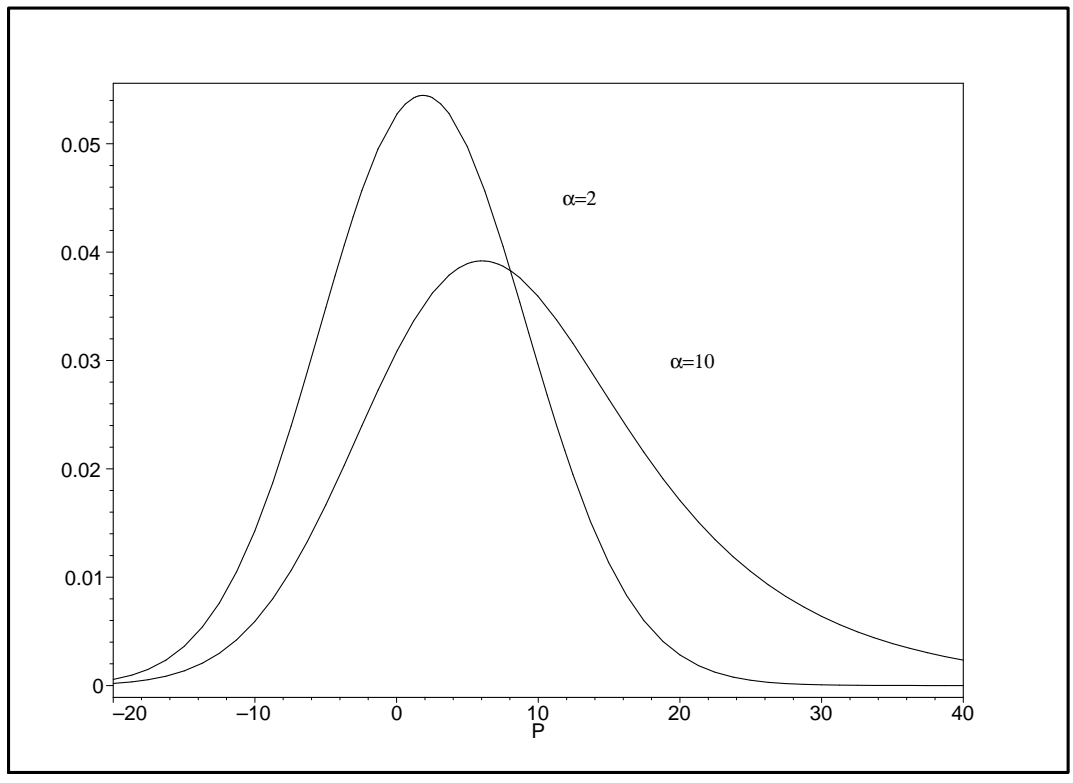

Figure 3: The pointer final wave function for the case of weak back-reaction. The initial pointer wave function is a Gaussian of width $\Delta=10$ centered around $P=0$. The shifts in the two cases here are $\alpha=2$ and $\alpha=10$. In the latter case the non-symmetric distortion indicates an increase back-reaction. 
The behavior of the pointer in the first weak limit is exhibited in Fig. 3. for different values of $\alpha<\Delta$. In this weak limit a single measurement is unable to resolve between the observable eigenvalues. When the measurement is repeated many times the average shift will tend to the expectation value of $J$. However in each trail we cannot distinguish between different eigenvalues.

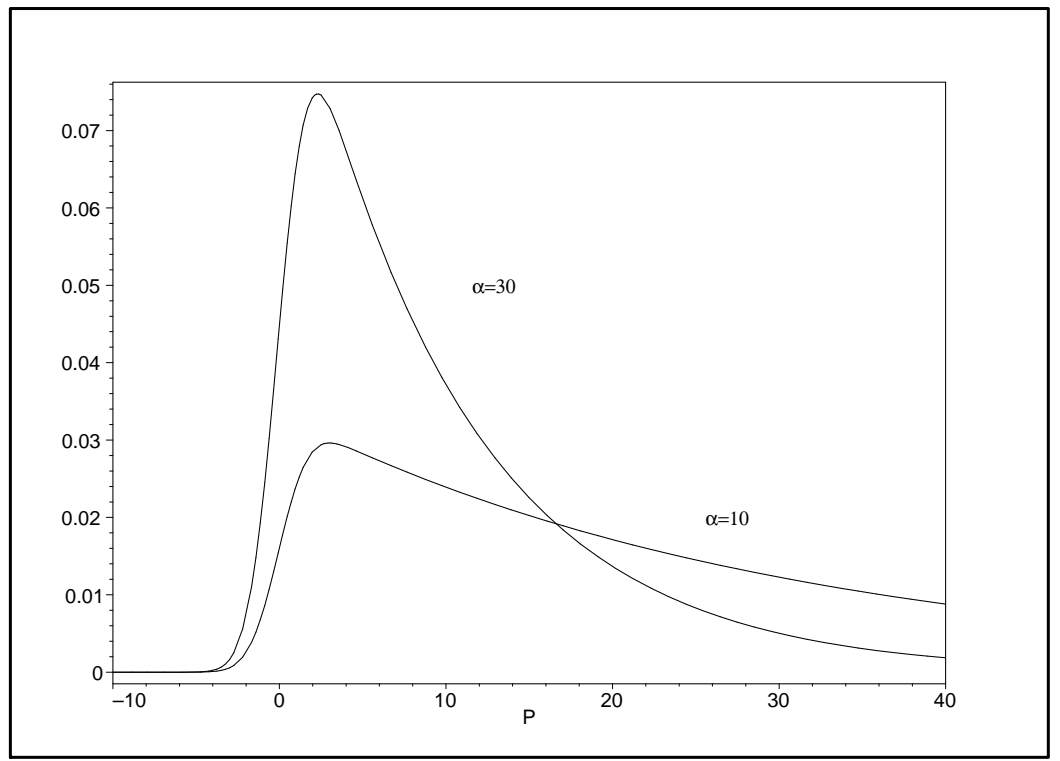

Figure 4: The pointer's final wave function for the case of strong backreaction, occurring when the measurement becomes accurate $\Delta<\alpha$. Here the resolution is fixed as $\Delta=5$ and $\alpha=10,20,30$

Next let us examine the "strong" limit, exhibited in Fig. 4., when due to the back-reaction the major part of the clock wave function is reflected back and $|T| \ll 1$. This corresponds to the case case $\alpha \gg \Delta$, of high resolution. We find

$$
|\Psi\rangle_{T} \simeq \int d k \phi_{C}(k) \sum_{j} C_{j} e^{-\frac{k}{M}\left|\frac{P}{j}\right|} \theta(P j)|k\rangle|P\rangle|j\rangle
$$

If for instance we find $P=P_{0}>0$ this reduces ("after" the measurement) to

$$
\left|P_{0}\right\rangle \sum_{j>0} C_{j}\left|\phi_{j}\right\rangle|j\rangle
$$


where

$$
\left|\phi_{j}\right\rangle=\int d k e^{-\frac{k}{M}\left|\frac{P_{0}}{j}\right|} \phi_{C}(k)|k\rangle
$$

The state of the system hence remains in a mixed state. Notice that the states $\left|\phi_{j}\right\rangle$ are not an orthogonal set of clock states. Hence by further measuring (post-selecting) the state of the clock we cannot reduce the system into one of the eigenstates $j$.

To summarize, in the impulsive limit, none of the cases examined above can realize an ordinary measurement. In the weak limit, when the back reaction is small, we can observe statistically the expectation value of the observable, but cannot resolve eigenvalues. In the strong back-reaction limit, corresponding to an accurate measurement, again we did not resolve the eigenvalues, and the final state of the system remains entangled with that of the clock. A further measurement on the clock does not help to reduce the system to a definite pure state.

\section{Discussion}

The harmony between the fundamental postulates of quantum mechanics and von-Neumann's measurement theory, breaks down if a physical clock, with bounded energy, is used as a timing device for the measurement. We have seen that to avoid back-reaction the accuracy of the measurement must be limited and is bounded by $\hbar / E_{C} \Delta T$. Hence, the energy of the clock sets up a scale of minimal time $\hbar / E_{C}$. Only for observables which evolve slowly in that time does the ordinary the theory of measurement apply with small corrections. For other cases a measurement does not "reduce" the state to a pure state corresponding to an eigenvalue of the observable. Therefore the state of a sub-system of a closed system, may not be observed in the usual sense. Perhaps this indicates that the quantization of closed systems must incorporate mixed states on a fundamental level.

Acknowledgment We thank Yakir Aharonov for many helpful discussions. B. R. acknowledges the support from grant 614/95 of the Israel Science Foundation, established by the Israel Academy of Sciences and Humanities. 


\section{A Appendix: Free particle clock}

In this appendix we will review the basic properties of the free particle toy model used as a clock in this article, with the Hamiltonian

$$
H_{C}=\frac{P_{X}^{2}}{2 M}
$$

as is well known one cannot construct an exact (conjugate) time operator for a bounded Hamiltonian which is also self adjoint. Instead we consider an approximate time operator define by

$$
\tau=\frac{X}{\left(\left\langle P_{X}\right\rangle / M\right)}
$$

where $\left\langle P_{X}\right\rangle$ is the expectation value of $P_{X}$. We have

$$
\left[\tau, H_{C}\right]=i \frac{P}{\left\langle P_{X}\right\rangle}=i+O\left(i \frac{\Delta P_{X}}{\left\langle P_{X}\right\rangle}\right)
$$

If the clock state are chosen to satisfy $\Delta P / P \ll 1$ the right hand side can be made close to $i$.

Let us examine the requirements needed from a clock for a specific initial Gaussian state. The degree to which this clock can approximate an idealclock is defined by its accuracy and by the duration it is usable. The accuracy of the clock is defined by how close can we approximate the ideal Newtonian time $t$, by the physical time $\tau$. The usable time, is determined by the maximal time interval that the uncertainty $\Delta \tau$ does not grow beyond the desired accuracy.

For the free particle clock, $\tau$ deviates from the ideal time $t$ by the uncertainty

$$
\Delta \tau \approx \frac{\Delta X(t)}{\left(\left\langle P_{X}\right\rangle / M\right)} \approx \frac{\Delta X(0)}{\left(\left\langle P_{X}\right\rangle / M\right)} \sqrt{1+\tau^{2} / M^{2} \Delta X^{4}(0)}
$$

The usable time interval is hence

$$
\tau_{\text {usable }} \approx M(\Delta X)^{2}
$$

or

$$
\frac{\tau_{\text {usable }}}{\Delta \tau} \approx \frac{\bar{P}^{2}}{M} \Delta \tau \approx \frac{\bar{E}}{\Delta E}
$$


Obviously we would like the left hand side to be much larger than unity. As we see this can be obtained for states with $E \gg \Delta E$, or alternatively, this implies $\bar{P} \gg \Delta P$ and $\Delta X \gg 1 / P=\lambda_{d e-B r o l i e}$. The latter conditions are identical to the conditions following from equation (40) 


\section{References}

[1] W. Pauli, in Encyclopedia of physics, eds. S. Flugee, , Vol. 5 Part 1, (Berlin, Springer Verlag, 1958).

[2] K.V. Kuchar, 1992, Proceeding of the 4th Canadian Conference on General Relativity and Relativistic Astrophysics, Ed. by G. Kunstatter, D. Vincent and J Williams (World Scientific) Singapore.

[3] J. B, Hartle, Phys. Rev. D38, 2985 (1988).

[4] W. G. Unruh and R. M. Wald, Phys. Rev. D40 2598 (1989).

[5] D. Page and W. Wootters, Phys. Rev. D27, 2885 (1983).

[6] F. Englert, Phys. Lett. B334, 472, (1990).

[7] J. Oppenheim, B. Reznik, W. G. Unruh, "Time as an Ovservable", Proceeding of the 10th Max Born Symposium, eds Ph. Blachard, A. Jadczyk, Worclow, Sept. 1997, Springer-Verlag, Lecture Notes in Physics.

[8] J. von Neumann, Mathematical Foundations of Quantum Mechanics, Princeton University Press, Princeton (1955).

[9] A. Peres, Am. J. Phys. 48, 552 (1980).

[10] Y. Aharonov, J. Oppenheim, S. Popescu, B. Reznik and W. G. Unruh, Phys. Rev. A57, 4130 (1988). quant-ph/9709031.

[11] Y. Aharonov, and B. Reznik, "Weiging a closed system and the timeenergy uncertainty priciple", quant-ph/9906030.

[12] T. Banks, Nucl. Phys. B249, 332 (1985).

[13] A. Casher and F. Englert, Class. and Quant. Grav. 9, 223 (1992).

[14] C. Rovelli, Phys. Rev. D 42 2638, (1990).

[15] C. Rovelli, Phys. Rev. D 43442 (1992).

[16] Y. Aharonov and L. Susskind, phys. Rev., 155, 1428 (1967).

[17] G. R. Allcock, Ann. Phys. 53288 (1969). 